\title{
Solution of the Dirac equation in the near horizon geometry of an extreme Kerr black hole
}

\author{
I. Sakalli* and M. Halilsoy ${ }^{\dagger}$ \\ Physics Department, EMU G.Magosa, Mersin 10, Turkey
}

(Received 15 January 2004; published 14 June 2004)

\begin{abstract}
The Dirac equation is solved in the near horizon limit geometry of an extreme Kerr black hole. We decouple equations first, as usual, into an axial and angular part. The axial equation turns out to be independent of the mass and is solved exactly. The angular equation reduces, in the massless case, to a confluent Heun equation. In general for a nonzero mass, the angular equation is expressible, at best, as a set of coupled first order differential equations apt for numerical investigation. The axial potentials corresponding to the associated Schrödinger-type equations and their conserved currents are found. Finally, based on our solution, we verify in a similar way the absence of superradiance for Dirac particles in the near horizon, a result which is well known within the context of a general Kerr background.
\end{abstract}

DOI: 10.1103/PhysRevD.69.124012

PACS number(s): 04.20.Jb

\section{INTRODUCTION}

During the last three decades the study of spin- $-\frac{1}{2}$ particles on type-D spacetimes has attracted much interest and by now accumulated results are already available in the literature $[1-3]$ (and references cited therein). The main reason for this is that all well-known black holes (BHs) are in this category and their better understanding involves a detailed analysis of various physical fields in their vicinity. Dirac particles with (and without) mass constitute one such potential candidate whose interaction and behavior around BHs may reveal information of much significance. Tests of the Dirac equation in spacetimes other than BHs have also started to arouse interest for various reasons. From this token we cite the Robertson-Walker and Bertotti-Robinson (BR) spacetimes [4-6]. The latter in particular has already gained much recognition in connection with extremal BHs, higher dimensions, and the brane world.

In this paper we consider the Dirac equation in the near horizon geometry of an extreme Kerr $\mathrm{BH}$. This constitutes the most important region of the outerworld prior to the horizon of a Kerr BH enhanced with the extremality condition. Extremal BHs are believed to have connection with the ground states of quantum gravity. This alone justifies, in spite of the absence of backreaction effects, the importance of spin- $\frac{1}{2}$ particles on such backgrounds. The throat geometry is a completely regular vacuum solution with an enhanced symmetry group $S L(2, R) \times U(1)$. In many aspects, this solution shares common features with the $\mathrm{AdS}_{2} \times S_{2}$ geometry arising in the near horizon limit of extreme ReissnerNordström BHs. The behavior of massless scalar fields in the extreme Kerr throat has been considered, and it is found that certain modes with large azimuthal quantum number exhibit superradiance [7]. This implies that the geodesics near the horizon can escape to infinity carrying energy-momentum more than the amount that infalls. Our solution enables us to investigate a similar phenomenon with Dirac fields which turns out to be negative as far as superradiance is conserved.

\footnotetext{
*Email address: izzet.sakalli@emu.edu.tr

${ }^{\dagger}$ Email address: mustafa.halilsoy@emu.edu.tr
}

This result is in accordance with the treatment of the Dirac equation in the general Kerr background in the absence of an exact solution [1]. The analysis of fermions in the KerrNewman background and the absence of superradiance was shown first by Lee [3]. Our aim is to reexamine this itemnot only by separating equations and deducing results on general grounds-but rather obtaining exact solutions and employing them. The advantage of confining ourselves to the near horizon alone bears fruits by allowing solutions expressible in terms of known polynomials. In the general background of the Kerr family of BHs even this much remains a problem beyond technical reach. Meanwhile, it is important to note that in contrast to the general Kerr metric, the throat metric is not asymptotically flat. This difference naturally shows itself in the potentials, too. In other words, the extreme Kerr throat metric represents such a local region that the geometry of interest is different than the geometry of the general Kerr metric. The steeply rising potential prevents any particle (or field) flow to infinity to make superradiance. Hence, we can mentally say that the behavior of particles in two geometries must be considered separately.

In order to separate equations we employ the well-known method due to Chandrasekhar so that we prefer to label the set of equations as Chandrasekhar-Dirac (CD) equations. We separate the $y$ (axial) and $\theta$ (angular) dependence in such a way that the resulting axial equation remains independent of mass. This leads us to an exact solution irrespective of mass. The angular equation on the other hand depends strictly on the mass. For the massless case (which we refer to as a neutrino equation) the angular equation reduces to a confluent Heun equation [8]. When the mass is nonzero, however, we cannot identify our equations but instead we express them as a set of linear equations suitable for numerical analysis.

The organization of the paper is as follows: in Sec. II we review the near horizon geometry of an extreme Kerr BH and separation of variables of the $\mathrm{CD}$ equation. Solution of the axial equation follows in Sec. III. The massless and massive cases are discussed in Secs. IV and V, respectively. The reduction of our equations to one-dimensional Schrödingertype equations with their conserved currents and superradi- 
ance is included in Sec. VI. The paper ends with a conclusion in Sec. VII.

\section{EXTREME KERR THROAT GEOMETRY AND SEPARATION OF DIRAC EQUATION ON IT}

The extreme Kerr metric in the Boyer-Lindquist coordinates is given by

$$
d s^{2}=e^{2 \nu} d \widetilde{t}^{2}-e^{2 \psi}(d \widetilde{\phi}+\omega d \tilde{t})^{2}-\rho^{2}\left(\frac{d \widetilde{r}^{2}}{\widetilde{\Delta}}+d \theta^{2}\right),
$$

where

$$
\begin{aligned}
e^{2 \nu} & =\frac{\widetilde{\Delta} \rho^{2}}{\left(\tilde{r}^{2}+M^{2}\right)^{2}-\widetilde{\Delta} M^{2} \sin ^{2} \theta}, \\
e^{2(\nu+\psi)} & =\widetilde{\Delta} \sin ^{2} \theta, \\
\tilde{\Delta} & =(\tilde{r}-M)^{2}, \\
\omega & =\frac{2 M^{2} \tilde{r} e^{2 \nu}}{\widetilde{\Delta} \rho^{2}}, \\
\rho^{2} & =\widetilde{r}^{2}+M^{2} \cos ^{2} \theta .
\end{aligned}
$$

In the extreme case, both the total mass $M$ and the rotation parameter $a$ become identical so that the angular momentum $J=M^{2}$ and the extremal horizon corresponds to $\tilde{r}=M$. The area of the horizon is $A=8 \pi J$.

To describe the near horizon (or throat) limit of the extreme Kerr metric, due originally to Bardeen and Horowitz [7], one can set

$$
\begin{aligned}
& \tilde{r}=M+\lambda r, \\
& \tilde{t}=\frac{t^{\prime}}{\lambda}, \\
& \widetilde{\phi}=\phi-\frac{t^{\prime}}{2 \lambda M},
\end{aligned}
$$

and take the limit $\lambda \rightarrow 0$. In these new coordinates, the throat metric is obtained as

$$
\begin{aligned}
d s^{2}= & F\left[\frac{r^{2}}{r_{0}^{2}} d t^{\prime 2}-\frac{r_{0}^{2}}{r^{2}} d r^{2}-r_{0}^{2} d \theta^{2}\right] \\
& -\frac{r_{0}^{2} \sin ^{2} \theta}{F}\left(d \phi+\frac{r}{r_{0}^{2}} d t^{\prime}\right)^{2},
\end{aligned}
$$

where

$$
\begin{aligned}
F & =\frac{1+\cos ^{2} \theta}{2}, \\
r_{0}^{2} & =2 M^{2} .
\end{aligned}
$$

We set further, for simplicity, $r_{0}^{2}=1$. This throat spacetime no longer has asymptotic flatness.
Finally, passing to more general coordinates,

$$
\begin{aligned}
y & =\frac{1}{2 r}\left[r^{2}\left(1+t^{\prime 2}\right)-1\right], \\
\cot t & =\frac{1}{2 t^{\prime} r}\left[r^{2}\left(1-t^{\prime 2}\right)+1\right], \\
\phi & =\varphi+\ln \left|\frac{\cos t+y \sin t}{1+t^{\prime} r}\right|,
\end{aligned}
$$

we can write the throat metric (4) as

$$
d s^{2}=F\left[\left(1+y^{2}\right) d t^{2}-\frac{d y^{2}}{1+y^{2}}-d \theta^{2}\right]-\frac{\sin ^{2} \theta}{F}(d \varphi+y d t)^{2}
$$

The metric functions in Eq. (7) depend only on the variable $\theta$ and thus as expected in the search for a solution of the Dirac equation the angular equation forms the crux of the problem. The coordinates $-\infty<t<\infty,-\infty<y<\infty$ cover the entire, singularity free spacetime. The Killing vector $\partial / \partial t$ is not timelike everywhere; it admits a region (for $\sin ^{2} \theta$ $>0.536)$ in which it becomes spacelike. Therefore by a coordinate transformation this particular region is transformable into the spacetime of colliding plane waves [9]. Recently, it has also been shown that the metric (7) can be obtained as a solution to dilaton-axion gravity which is similar to the rotating BR spacetime [10].

The singularity free character can best be seen by checking the Weyl scalar $\Psi_{2}$ and the Kretschmann scalar:

$$
\begin{aligned}
& \Psi_{2}=\frac{2}{\left(1+\cos ^{2} \theta\right)^{3}}\left[3 \cos ^{2} \theta-1+i \cos \theta\left(\cos ^{2} \theta-3\right)\right], \\
& R_{\mu \nu \rho \sigma} R^{\mu \nu \rho \sigma}=\frac{192 \sin ^{2} \theta}{\left(1+\cos ^{2} \theta\right)^{6}}\left[\left(1+\cos ^{2} \theta\right)^{2}-16 \cos ^{2} \theta\right] .
\end{aligned}
$$

When the backreaction of the spin- $\frac{1}{2}$ test particles on the background geometry is neglected, the Dirac field equation is given by the $\mathrm{CD}$ equations [1] on a fixed spacetime (7).

We choose a complex null tetrad $\{l, n, m, \bar{m}\}$ that satisfies the orthogonality conditions $l \cdot n=-m \cdot \bar{m}=1$. We note that, throughout the paper, an overbar denotes complex conjugation. Thus the covariant one-forms can be written as

$$
\begin{aligned}
& \sqrt{2} l=\sqrt{F\left(1+y^{2}\right)} d t-\frac{\sqrt{F}}{\sqrt{1+y^{2}}} d y, \\
& \sqrt{2} n=\sqrt{F\left(1+y^{2}\right)} d t+\frac{\sqrt{F}}{\sqrt{1+y^{2}}} d y,
\end{aligned}
$$




$$
\begin{aligned}
& \sqrt{2} m=\frac{i y \sin \theta}{\sqrt{F}} d t+\sqrt{F} d \theta+\frac{i \sin \theta}{\sqrt{F}} d \varphi, \\
& \sqrt{2} \bar{m}=-\frac{i y \sin \theta}{\sqrt{F}} d t+\sqrt{F} d \theta-\frac{i \sin \theta}{\sqrt{F}} d \varphi,
\end{aligned}
$$

and their corresponding directional derivatives are

$$
\begin{aligned}
& \sqrt{2} D=\frac{1}{\sqrt{F\left(1+y^{2}\right)}} \partial_{t}+\frac{\sqrt{1+y^{2}}}{\sqrt{F}} \partial_{y}, \\
& \sqrt{2} \Delta=\frac{1}{\sqrt{F\left(1+y^{2}\right)}} \partial_{t}-\frac{\sqrt{1+y^{2}}}{\sqrt{F}} \partial_{y}, \\
& \sqrt{2} \delta=-\frac{1}{\sqrt{F}} \partial_{\theta}-\frac{i \sqrt{F}}{\sin \theta} \partial_{\varphi}, \\
& \sqrt{2} \bar{\delta}=-\frac{1}{\sqrt{F}} \partial_{\theta}+\frac{i \sqrt{F}}{\sin \theta} \partial_{\varphi} .
\end{aligned}
$$

One can determine the nonzero Newman-Penrose (NP) complex spin coefficients [11] in the above null tetrad as

$$
\begin{aligned}
& \pi=-\tau=\frac{\sin \theta(\cos \theta-i)}{(2 F)^{3 / 2}}, \\
& \varepsilon=\gamma=\frac{y}{2 \sqrt{2 F\left(1+y^{2}\right)}}, \\
& \alpha=-\beta=\frac{2 \cot \theta-i \sin \theta}{2(2 F)^{3 / 2}} .
\end{aligned}
$$

The $\mathrm{CD}$ equations in the NP formalism are then [1]

$$
\begin{gathered}
(D+\varepsilon-\rho) F_{1}+(\bar{\delta}+\pi-\alpha) F_{2}=i \mu_{p} G_{1}, \\
(\Delta+\mu-\gamma) F_{2}+(\delta+\beta-\tau) F_{1}=i \mu_{p} G_{2}, \\
(D+\bar{\varepsilon}-\bar{\rho}) G_{2}-(\delta+\bar{\pi}-\bar{\alpha}) G_{1}=i \mu_{p} F_{2}, \\
(\Delta+\bar{\mu}-\bar{\gamma}) G_{1}-(\bar{\delta}+\bar{\beta}-\bar{\tau}) G_{2}=i \mu_{p} F_{1},
\end{gathered}
$$

where $\mu^{*}=\sqrt{2} \mu_{p}$ is the mass of the Dirac particle. $[1,6]$

The form of the $\mathrm{CD}$ equations suggests that we introduce

$$
\begin{aligned}
& F_{1}=f_{1}(y) A_{1}(\theta) e^{i(\sigma t+m \varphi)}, \\
& G_{1}=g_{1}(y) A_{2}(\theta) e^{i(\sigma t+m \varphi)}, \\
& F_{2}=f_{2}(y) A_{3}(\theta) e^{i(\sigma t+m \varphi)},
\end{aligned}
$$

$$
G_{2}=g_{2}(y) A_{4}(\theta) e^{i(\sigma t+m \varphi)},
$$

where $\sigma$ is the frequency of the corresponding Compton wave of the Dirac particle and $m$ is the azimuthal quantum number of the wave. Our convention is that $\sigma$ is always positive.

Inserting for the appropriate spin coefficients (12) with the spinors (14) into the four coupled CD equations (13), we obtain

$$
\begin{aligned}
\frac{\left(\widetilde{Z} f_{1}\right)}{f_{2}} & =i \mu * \frac{g_{1}}{f_{2}} \frac{A_{2}}{A_{1}} \sqrt{F}-\frac{\left(L A_{3}\right)}{A_{1}}, \\
\frac{\left(\bar{Z} f_{2}\right)}{f_{1}} & =-i \mu * \frac{g_{2}}{f_{1}} \frac{A_{4}}{A_{3}} \sqrt{F}-\frac{\left(L^{+} A_{1}\right)}{A_{3}}, \\
\frac{\left(\widetilde{Z} g_{2}\right)}{g_{1}} & =i \mu * \frac{f_{2}}{g_{1}} \frac{A_{3}}{A_{4}} \sqrt{F}-\frac{\left(£^{+} A_{2}\right)}{A_{4}}, \\
-\frac{\left(\bar{Z} g_{1}\right)}{g_{2}} & =i \mu * \frac{f_{1}}{g_{2}} \frac{A_{1}}{A_{2}} \sqrt{F}-\frac{\left(£ A_{4}\right)}{A_{2}},
\end{aligned}
$$

where the axial and the angular operators are

$$
\begin{aligned}
& \widetilde{Z}=\sqrt{1+y^{2}} \partial_{y}+\frac{1}{2 \sqrt{1+y^{2}}}[y+2 i \sigma], \\
& \bar{Z}=\sqrt{1+y^{2}} \partial_{y}+\frac{1}{2 \sqrt{1+y^{2}}}[y-2 i \sigma],
\end{aligned}
$$

and

$$
\begin{aligned}
& L=\partial_{\theta}+\frac{m F}{\sin \theta}+\frac{1}{2 F}\left(\frac{\cos ^{3} \theta}{\sin \theta}+\frac{i \sin \theta}{2}\right), \\
& L^{+}=\partial_{\theta}-\frac{m F}{\sin \theta}+\frac{1}{2 F}\left(\frac{\cos ^{3} \theta}{\sin \theta}+\frac{i \sin \theta}{2}\right), \\
& £=\partial_{\theta}+\frac{m F}{\sin \theta}+\frac{1}{2 F}\left(\frac{\cos ^{3} \theta}{\sin \theta}-\frac{i \sin \theta}{2}\right), \\
& £^{+}=\partial_{\theta}-\frac{m F}{\sin \theta}+\frac{1}{2 F}\left(\frac{\cos ^{3} \theta}{\sin \theta}-\frac{i \sin \theta}{2}\right),
\end{aligned}
$$

respectively. One can easily see that $L=\bar{£}$ and $L^{+}=\overline{£^{+}}$.

Further, choosing $f_{1}=g_{2}, f_{2}=g_{1}, A_{1}=\overline{A_{2}}$, and $A_{3}=\overline{A_{4}}$ and introducing the separation constant as $i \lambda$, where $\lambda$ is a real constant, we can separate Dirac equation (15) into axial and angular parts

$$
\begin{aligned}
& \overline{\widetilde{Z}} g_{1}=-i \lambda g_{2}, \\
& \widetilde{Z} g_{2}=i \lambda g_{1}
\end{aligned}
$$


and

$$
\begin{gathered}
L A_{3}+i \mu^{*} A_{2} \sqrt{F}=i \lambda A_{1}, \\
L^{+} A_{1}+i \mu^{*} A_{4} \sqrt{F}=i \lambda A_{3} .
\end{gathered}
$$

It is clear from Eqs. (18), (19) that $g_{1}=\bar{g}_{2}$.

\section{SOLUTION OF THE AXIAL EQUATION}

If we decouple the axial equations (18), (19) in Eq. (18) to get $g_{1}$, we obtain

$$
\widetilde{Z}\left(\overline{\widetilde{Z}} g_{1}\right)=\lambda^{2} g_{1}
$$

Similarly one can decouple the axial equations in Eq. (18) for $g_{2}$. The explicit form of Eq. (22) can be obtained as

$$
\begin{aligned}
& \left(1+y^{2}\right) g_{1}^{\prime \prime}(y)+2 y g_{1}^{\prime}(y) \\
& +\frac{1}{1+y^{2}}\left(\frac{1}{2}+\sigma^{2}+\frac{y^{2}}{4}-\lambda^{2}\left(1+y^{2}\right)+i \sigma y\right) g_{1}(y)=0 .
\end{aligned}
$$

(Throughout the paper, a prime denotes a derivative with respect to its argument.)

Thus the solutions of the decoupled equations for $g_{1}$, Eq. (23), and $g_{2}$ (not given here) can be found in terms of the associated Legendre functions as follows:

$$
\begin{aligned}
& g_{1}(y)=c_{1} P_{\lambda-1 / 2}^{\hat{\beta}}(i y), \\
& g_{2}(y)=c_{2} P_{\lambda-1 / 2}^{\hat{\beta}}(-i y),
\end{aligned}
$$

where

$$
\hat{\beta}=\sqrt{\sigma^{2}+\frac{\sigma}{2}+\frac{1}{4}}
$$

and $c_{1}, c_{2}$ are complex constants.

Here, as a result of the physical necessities, we considered only the first kind of the associated Legendre functions. Although solutions (24) seem like complex solutions, it is possible to draw real functions from the above associated Legendre functions. We may define

$$
\lambda=\tilde{m}+\frac{1}{2} \text { with } \tilde{m}=1,2,3, \ldots
$$

and

$$
\sigma=\frac{1}{4}\left(\sqrt{16 \tilde{n}^{2}-3}-1\right)
$$

so that

$$
\hat{\beta}=\tilde{n} \quad \text { with } \quad \tilde{n}=-\tilde{m},-\tilde{m}+1, \ldots,-1,1, \ldots, \tilde{m}-1, \tilde{m} .
$$

In order to get the real functions for solutions (22), the required condition is $\tilde{m}-|\tilde{n}|=$ even number.

It is worth also drawing attention to the following remarks.

(i) In the case of $\lambda=0$, Eqs. (18), (19) reduce to simple first order differential equations which admit the solutions

$$
\begin{aligned}
& g_{1}(y)=c_{3}\left(1+y^{2}\right)^{-1 / 4} e^{i \sigma \tan ^{-1}(y)}, \\
& g_{2}(y)=c_{4}\left(1+y^{2}\right)^{-1 / 4} e^{-i \sigma \tan ^{-1}(y)},
\end{aligned}
$$

with $c_{3}, c_{4}$ complex constants.

These two solutions can be interpreted as representing ingoing and outgoing waves.

(ii) In the case of $\lambda=\frac{1}{2}$, we obtain the following complex solutions from Eqs. (18), (19):

$$
\begin{aligned}
& g_{1}(y)=c_{5}\left(\frac{i y+1}{i y-1}\right)^{\hat{\beta} / 2}+c_{6}\left(\frac{i y+1}{i y-1}\right)^{-\hat{\beta} / 2}, \\
& g_{2}(y)=c_{7}\left(\frac{1-i y}{1+i y}\right)^{\hat{\beta} / 2}+c_{8}\left(\frac{1-i y}{1+i y}\right)^{-\hat{\beta} / 2},
\end{aligned}
$$

where again $c_{j}$ with $j=5,6,7,8$ are complex constants.

\section{REDUCTION OF THE ANGULAR EQUATION TO THE HEUN EQUATION: THE MASSLESS CASE}

In this section, we shall show that the angular equations (20), (21) for the neutrino particles can be decoupled to the confluent Heun equation. To the end that let us reconsider Eqs. (20), (21) in an explicit form for $\mu^{*}=0$ :

$$
\begin{aligned}
& A_{3}^{\prime}(\theta)+(K+G) A_{3}(\theta)=i \lambda A_{1}(\theta), \\
& A_{1}^{\prime}(\theta)+(K-G) A_{1}(\theta)=i \lambda A_{3}(\theta),
\end{aligned}
$$

where

$$
\begin{gathered}
K=\frac{1}{2 F}\left(\frac{\cos ^{3} \theta}{\sin \theta}+\frac{i \sin \theta}{2}\right), \\
G=\frac{m F}{\sin \theta} .
\end{gathered}
$$

By introducing the scalings

$$
\begin{aligned}
& A_{1}(\theta)=H_{1}(\theta) \exp \left(-\int(K-G) d \theta\right), \\
& A_{3}(\theta)=H_{3}(\theta) \exp \left(-\int(K+G) d \theta\right),
\end{aligned}
$$

one gets

$$
H_{1}^{\prime}(\theta)=i \lambda H_{3}(\theta) \exp \left(-\int 2 G d \theta\right)
$$




$$
H_{3}^{\prime}(\theta)=i \lambda H_{1}(\theta) \exp \left(\int 2 G d \theta\right) \text {. }
$$

If we decouple Eqs. (37), (38) in Eq. (37) for $H_{1}(\theta)$, we get

$$
H_{1}^{\prime \prime}(\theta)+2 G H_{1}^{\prime}(\theta)+\lambda^{2} H_{1}(\theta)=0 .
$$

In similar fashion, we find, for $H_{3}(\theta)$,

$$
H_{3}^{\prime \prime}(\theta)-2 G H_{3}^{\prime}(\theta)+\lambda^{2} H_{3}(\theta)=0 \text {. }
$$

Introducing a new variable $\theta=\cos ^{-1}(1-2 z)$, Eqs. (39), (40) turn out to be

$$
\begin{aligned}
& H_{1}^{\prime \prime}(z)+\left(-2 m+\frac{\frac{1}{2}+m}{z}+\frac{\frac{1}{2}-m}{z-1}\right) H_{1}^{\prime}(z) \\
& -\frac{\lambda^{2}}{z(z-1)} H_{1}(z)=0, \\
& H_{3}^{\prime \prime}(z)+\left(-2 m+\frac{m-\frac{1}{2}}{z}+\frac{m+\frac{1}{2}}{z-1}\right) H_{3}^{\prime}(z) \\
& -\frac{\lambda^{2}}{z(z-1)} H_{3}(z)=0 .
\end{aligned}
$$
$[8]$

Let us recall the general confluent form of Heun equation

$$
H^{\prime \prime}(z)+\left(A+\frac{B}{z}+\frac{C}{z-1}\right) H^{\prime}(z)-\frac{D B z-h}{z(z-1)} H(z)=0 .
$$

Drawing the similarities between Eq. (43) and Eqs. (41), (42), we observe the following correspondences.

(a) For Eq. (41),

$$
D=0, \quad h=\lambda^{2}, \quad A=-2 m, \quad B=\frac{1}{2}+m, \quad C=\frac{1}{2}-m .
$$

(b) For Eq. (42),

$$
D=0, \quad h=\lambda^{2}, \quad A=-2 m, \quad B=m-\frac{1}{2}, \quad C=m+\frac{1}{2} .
$$

The confluent Heun equation (43), with its accessory parameter $h$, has two regular singular points at $z=0,1$ with exponents $(0,1-B)$ and $(0,1-C)$, respectively, as well as an irregular singularity at infinity. In the vicinity of the point $z$ $=0$, its power series can be written as

$$
H(D, A, B, C, h ; z)=\sum_{j=0}^{\infty} W_{j} z^{j}
$$

and the coefficient $W_{j}$ satisfies a three-term recurrence relation [8]

$$
\begin{gathered}
W_{0}=1, \quad W_{1}=\frac{-h}{B}, \\
(j+1)(j+B) W_{j+1}-A(j-1+D) W_{j-1} \\
=[j(j-1-A+B+C)-h] W_{j} .
\end{gathered}
$$

It is also possible to obtain the power series solution in the vicinity of the point $z=1$ by a linear transformation interchanging the regular singular points $z=0$ and $z=1$. Namely, $z \rightarrow 1-z$.

Expansion of solutions to the confluent Heun equation in terms of the hypergeometric and confluent hypergeometric functions can be seen in [8]. In Ref. [8], it is also shown that the confluent Heun equation can be normalized to constitute a group of orthogonal complete functions and the confluent Heun equation also admits quasipolynomial solutions for particular values of the parameters.

Since $D=0$ in our case, it follows from the three-term recurrence relation that $H(D, A, B, C, h ; z)$ is a polynomial solution if $W_{1}(h)=0$, where $W_{1}$ stands for a polynomial of degree 1 in $h$. Namely, there is only one eigenvalue $h_{i}$ for $h$ such that $W_{1}\left(h_{i}\right)=0$ (i.e., $\left.\lambda=0\right)$.

\section{REDUCTION OF THE ANGULAR EQUATION INTO A SET OF LINEAR FIRST ORDER DIFFERENTIAL EQUATIONS: THE CASE WITH MASS}

To complete our analysis of the angular equation, we need to discuss the angular equation for the Dirac particles with mass. forms

The angular equations (20), (21) can be rewritten in the

$$
\begin{gathered}
L A_{3}+i \mu_{p} \sqrt{1+\cos ^{2} \theta} \overline{A_{1}}=i \lambda A_{1}, \\
L^{+} A_{1}+i \mu_{p} \sqrt{1+\cos ^{2} \theta} \overline{A_{3}}=i \lambda A_{3} .
\end{gathered}
$$

With substitutions

$$
\begin{aligned}
& A_{1}(\theta)=\left[A_{0}(\theta)+i B_{0}(\theta)\right] \exp \left(\int \frac{\cos ^{3} \theta}{2 \sin \theta F} d \theta\right), \\
& A_{3}(\theta)=\left[\left(M_{0}(\theta)+i N_{0}(\theta)\right] \exp \left(\int \frac{\cos ^{3} \theta}{2 \sin \theta F} d \theta\right),\right.
\end{aligned}
$$

we can transform Eqs. (48), (49) into a set of first order differential equations

$$
\begin{aligned}
& M_{0}^{\prime}(\theta)+G M_{0}(\theta)-\frac{\sin \theta}{4 F} N_{0}(\theta) \\
& =-\left(\lambda+\mu_{p} \sqrt{1+\cos ^{2} \theta}\right) B_{0}(\theta),
\end{aligned}
$$




$$
\begin{gathered}
N_{0}^{\prime}(\theta)+G N_{0}(\theta)+\frac{\sin \theta}{4 F} M_{0}(\theta) \\
=\left(\lambda-\mu_{p} \sqrt{1+\cos ^{2} \theta}\right) A_{0}(\theta), \\
A_{0}^{\prime}(\theta)-G A_{0}(\theta)-\frac{\sin \theta}{4 F} B_{0}(\theta) \\
=-\left(\lambda+\mu_{p} \sqrt{1+\cos ^{2} \theta}\right) N_{0}(\theta), \\
B_{0}^{\prime}(\theta)-G B_{0}(\theta)+\frac{\sin \theta}{4 F} A_{0}(\theta)=\left(\lambda-\mu_{p} \sqrt{1+\cos ^{2} \theta}\right) M_{0}(\theta) .
\end{gathered}
$$

Introducing a new variable $x=\cos \theta$ and with the further substitutions

$$
\begin{array}{ll}
M_{0}(\theta)=\frac{1}{2}\left[m_{0}(\theta)+a_{0}(\theta)\right], & N_{0}(\theta)=\frac{1}{2}\left[n_{0}(\theta)+b_{0}(\theta)\right], \\
A_{0}(\theta)=\frac{1}{2}\left[m_{0}(\theta)-a_{0}(\theta)\right], & B_{0}(\theta)=\frac{1}{2}\left[n_{0}(\theta)-b_{0}(\theta)\right],
\end{array}
$$

we may obtain the final form of the set as linear first order differential equations

$$
\begin{aligned}
& m_{0}^{\prime}(x)+\alpha_{1} a_{0}(x)+\left(\alpha_{2}-\alpha_{3}\right) n_{0}(x)=0, \\
& a_{0}^{\prime}(x)+\alpha_{1} m_{0}(x)+\left(\alpha_{4}+\alpha_{3}\right) b_{0}(x)=0, \\
& n_{0}^{\prime}(x)+\alpha_{1} b_{0}(x)-\left(\alpha_{2}+\alpha_{3}\right) m_{0}(x)=0, \\
& b_{0}^{\prime}(x)+\alpha_{1} n_{0}(x)-\left(\alpha_{4}-\alpha_{3}\right) a_{0}(x)=0,
\end{aligned}
$$

where

$$
\begin{gathered}
\alpha_{1}=-\frac{m\left(1+x^{2}\right)}{2\left(1-x^{2}\right)}, \quad \alpha_{2}=\frac{1}{2\left(1+x^{2}\right)}-\frac{\lambda}{\sqrt{1-x^{2}}}, \\
\alpha_{3}=\frac{\mu_{p} \sqrt{1+x^{2}}}{\sqrt{1-x^{2}}}, \quad \alpha_{2}=\frac{1}{2\left(1+x^{2}\right)}+\frac{\lambda}{\sqrt{1-x^{2}}} .
\end{gathered}
$$

Although the system (54) does not seem to be solved analytically, one may develop an appropriate numerical technique to study it. In the literature, there may exist such interesting systems which are more or less of this type.

\section{REDUCTION OF THE DIRAC EQUATION TO A ONE- DIMENSIONAL SCHRÖDINGER-TYPE EQUATION WITH CONSERVED CURRENT}

It is possible to get more compact forms the axial equations (18), (19) by introducing the scalings

$$
\begin{aligned}
& g_{1}(y)=Z_{1}(y)\left(1+y^{2}\right)^{-1 / 4}, \\
& g_{2}(y)=Z_{2}(y)\left(1+y^{2}\right)^{-1 / 4},
\end{aligned}
$$

and applying the coordinate transformation $y=\tan u$; the axial equations then take the form

$$
\begin{aligned}
& Z_{1}^{\prime}(u)-i \sigma Z_{1}(u)=-i \lambda X Z_{2}(u), \\
& Z_{2}^{\prime}(u)+i \sigma Z_{2}(u)=i \lambda X Z_{1}(u),
\end{aligned}
$$

where $X=\left(\sqrt{1+y^{2}}\right) \equiv 1 / \cos u$.

Letting

$$
\begin{aligned}
& Z_{1}(u)=\frac{i P_{1}(u)-P_{2}(u)}{2}, \\
& Z_{2}(u)=\frac{i P_{1}(u)+P_{2}(u)}{2},
\end{aligned}
$$

we can combine Eqs. (58), (59) to give

$$
\begin{gathered}
P_{1}^{\prime}(u)=-E_{+} P_{2}(u), \\
P_{2}^{\prime}(u)=E_{-} P_{1}(u),
\end{gathered}
$$

where

$$
\begin{aligned}
& E_{+}=\sigma+\lambda X, \\
& E_{-}=\sigma-\lambda X .
\end{aligned}
$$

Decoupling is attained by introducing

$$
\begin{aligned}
& P_{1}(u)=\sqrt{E_{+}} T(u), \\
& P_{2}(u)=\sqrt{E_{-}} S(u),
\end{aligned}
$$

where we obtain a pair of one-dimensional Schrödinger-type equations

$$
\begin{aligned}
& T^{\prime \prime}(u)+V_{1} T(u)=0, \\
& S^{\prime \prime}(u)+V_{2} S(u)=0,
\end{aligned}
$$

with the potentials

$$
\begin{aligned}
& V_{1}=\sigma^{2}-\lambda^{2} X^{2}\left(1+\frac{y^{2}}{4 E_{+}^{2}}\right)+\frac{\lambda X}{2 E_{+}^{2}}\left[\sigma\left(1+2 y^{2}\right)+\lambda X^{3}\right], \\
& V_{2}=\sigma^{2}-\lambda^{2} X^{2}\left(1+\frac{y^{2}}{4 E_{-}^{2}}\right)-\frac{\lambda X}{2 E_{-}^{2}}\left[\sigma\left(1+2 y^{2}\right)-\lambda X^{3}\right] .
\end{aligned}
$$

One can easily observe that for $y \rightarrow \pm \infty$ the potentials diverge. This result stems from the fact that our spacetime is not asymptotically flat.

To examine the existence of superradiance, one may consider the conserved net current of Dirac particles [1] - in other words, the rate $(\partial N / \partial t)_{i n}$ at which particles falling through the horizon per unit time, which must be negative for the superradiance to occur: 


$$
\left(\frac{\partial N}{\partial t}\right)_{\text {in }}=-\left.\left(\int \sqrt{-g} J^{y} d \theta d \varphi\right)\right|_{\text {horizon }}<0
$$

where $g$ is the determinant of the spacetime metric and $J^{y}$ is the axial component of the neutrino particle current. We recall from metric (7) that we have

$$
\sqrt{-g}=F \sin \theta
$$

It is clear from transformations (3), (6) that the horizon of metric (7) corresponds to $y \rightarrow(-\infty)$. In other words, integral (72) is taken over $y \rightarrow(-\infty)$. $[1]$

In the more standard spinor formalism, $J^{y}$ is introduced as

$$
\frac{1}{\sqrt{2}} J^{y}=\sigma_{A B^{\prime}}^{y}\left(P^{A} \bar{P}^{B^{\prime}}+Q^{A} \bar{Q}^{B^{\prime}}\right),
$$

where

$$
\sigma_{A B^{\prime}}^{y}=\frac{1}{\sqrt{2}}\left(\begin{array}{cc}
\sqrt{\frac{1+y^{2}}{F}} & 0 \\
0 & -\sqrt{\frac{1+y^{2}}{F}}
\end{array}\right) .
$$

In this notation, the basic spinors defined by $P^{A}$ and $\bar{Q}^{A}$ correspond to [6]

$$
\begin{gathered}
P^{0}=F_{1}, \quad P^{1}=F_{2}, \\
\bar{Q}^{0^{\prime}}=-G 2, \quad \bar{Q}^{1^{\prime}}=G 1 .
\end{gathered}
$$

We evaluate $J^{y}$ as

$$
J^{y}=\sqrt{\frac{1+y^{2}}{F}}\left(\left|g_{2}\right|^{2}-\left|g_{1}\right|^{2}\right)\left(\left|A_{1}\right|^{2}+\left|A_{3}\right|^{2}\right) .
$$

Assuming that the angular functions $A_{1}(\theta)$ and $A_{3}(\theta)$ are normalized to unity, the integral in Eq. (72) yields

$$
\int \sqrt{-g} J^{y} d \theta d \varphi=3.246 \pi\left(\left|Z_{2}\right|^{2}-\left|Z_{1}\right|^{2}\right) .
$$

From Eqs. (60), (61), (62), and (63), we successively find

$$
\begin{aligned}
\left|Z_{2}\right|^{2}-\left|Z_{1}\right|^{2} & =\frac{i}{2}\left(P_{1} \bar{P}_{2}-P_{2} \bar{P}_{1}\right) \\
& =\frac{-i}{2 E_{+}}\left[P_{1}, \bar{P}_{1}\right]_{u}
\end{aligned}
$$

where $\left[P_{1}, \bar{P}_{1}\right]_{u}$ is the Wronskian.

Therefore, in order to check the existence of superradiance, it will suffice to seek a solution for $P_{1}$ at the horizon.

The reality that the potentials $V_{1}$ and $V_{2}$ become infinite at both the horizon and $y \rightarrow \infty$ leads us to think of the problem as a problem of particles in an infinite potential well. Since the particles are bound inside the well, the principal physical fact requires that the solutions of the wave equations (68), (69) must be identically zero at the walls (the horizon and $y \rightarrow \infty)$. Clearly, the Wronskian vanishes at the horizon and it follows that the number of particles exiting the horizon per unit time is zero. Consequently, similar to the general Kerr background [1,3], there is also no superradiance in the extreme Kerr throat geometry.

\section{CONCLUSION}

Our aim in this paper was to do more than separating the Dirac equation in a sector of Kerr-namely, the extremal Kerr throat geometry and obtain exact solutions if possible. This premise has mostly been accomplished and it definitely will contribute to the wave mechanical aspects of spin- $\frac{1}{2}$ particles prior infalling into the extreme Kerr BH.

In the general Kerr background the radial Dirac equation was the harder part to be tackled compared with the angular part [1]. In the present problem of the extremal Kerr throat we have the opposite case: the axial part poses no more difficulty than the angular part does. For the massless case, we overcome the difficulty and attain an exact solution in terms of Heun polynomials. Inclusion of mass prevents this reduction and as a result we are unable to express the angular equation in terms of a set of known equations. This part of the problem can be handled numerically. Alternatively, the angular equation is cast into a pair of Schrödinger-type equations. Unlike the scalar field case Dirac fields exhibit no superradiance. The charge coupling of a Dirac particle to an extremal Kerr-Newman BH in its near horizon limit may reveal more information compared to the present case. This is the next stage of study that interests us.
[1] S. Chandrasekhar, The Mathematical Theory of Black Holes (Clarendon Press, London, 1983).

[2] E.G. Kalnins and W. Miller, Jr., J. Math. Phys. 33, 286 (1992).

[3] C.H. Lee, Phys. Lett. 68B, 152 (1977).

[4] A. Zecca, J. Math. Phys. 37, 874 (1996).

[5] S.O. Gilberto, Gen. Relativ. Gravit. 33, 395 (2001).

[6] I. Sakalli, Gen. Relativ. Gravit. 35, 1321 (2003).
[7] J. Bardeen and G.T. Horowitz, Phys. Rev. D 60, 104030 (1999).

[8] A. Ronveaux, Heun's Differential Equations (Oxford Science, Oxford, 1995).

[9] M. Halilsoy, "Near horizon geometry of extreme black holes and colliding waves," gr-qc/0210055.

[10] G. Clement and D. Gal'tsov, Nucl. Phys. B619, 741 (2001).

[11] E.T. Newman and R. Penrose, J. Math. Phys. 3, 566 (1962). 